OPEN ACCESS

Edited by:

Wei Hu,

Fudan University, China

Reviewed by:

Fei Gao,

University of Arkansas, United States

Si-Yang Huang,

Chinese Academy of Agricultural

Sciences, China

Hong-Juan Peng,

Southern Medical University, China

*Correspondence:

Junlong Zhao

zhaojunlong@mail.hzau.edu.cn

Specialty section:

This article was submitted to

Infectious Diseases,

a section of the journal

Frontiers in Microbiology

Received: 08 May 2017 Accepted: 20 November 2017

Published: 04 December 2017

Citation:

Pan M, Zhou Y, Wang Y, Li L, Song Y, Hou L and Zhao J (2017) Screening and Identification of the

Host Proteins Interacting with Toxoplasma gondii Rhoptry Protein ROP16. Front. Microbiol. 8:2408. doi: 10.3389/fmicb.2017.02408

\section{Screening and Identification of the Host Proteins Interacting with Toxoplasma gondii Rhoptry Protein ROP16}

\author{
Ming Pan', Yanqin Zhou ${ }^{1,2,3}$, Yifan Wang ${ }^{1}$, Longjiao $\mathrm{Li}^{1}$, Yongle Song ${ }^{1}$, Lun Hou ${ }^{1}$ and \\ Junlong Zhao ${ }^{1,2,3 *}$
}

${ }^{1}$ State Key Laboratory of Agricultural Microbiology, College of Veterinary Medicine, Huazhong Agricultural University, Wuhan, China, ${ }^{2}$ Key Laboratory for Development of Veterinary Diagnostic Products, Ministry of Agriculture, Huazhong Agricultural University, Wuhan, China, ${ }^{3}$ Cooperative Innovation Center for Sustainable Pig Production, Wuhan, China

Toxoplasma gondii, as a zoonotic protozoan parasite, develops sophisticated strategies to manipulate hosts for efficient intracellular survival. After successful invasion, T. gondii injects many effector proteins into host cells for various purposes. TgROP16 (T. gondii rhoptry protein 16), which is secreted from rhoptries into host cells, can activate the host STAT (signal transducer and activator of transcription) signaling pathway through phosphorylation of STAT3 and STAT6. However, whether there are other host proteins modulated by TgROP16 is currently unknown. In this study, yeast two-hybrid (Y2H) screen was used to look for additional host proteins interacting with TgROP16. Yeast cells expressing a mouse cDNA library cloned into the prey vector were used to mate with yeasts expressing ROP16 without signal peptide. Two mouse proteins, Dnaja1 (DnaJ heat shock protein family member A1) and Gabra4 (gamma-aminobutyric acid A receptor, subunit alpha 4) were identified to interact with ROP16 from this screen. Further analysis suggested that the Predomain of ROP16 played key roles in mediating interactions with these host proteins, whereas the contribution from the Kinase domain was minor. The interactions between Dnaja1 and different parts of ROP16 were also estimated in vivo by co-immunoprecipitation. The results showed that the Predomain of ROP16 was the major region to interact with Dnaja1, which is consistent with the $\mathrm{Y} 2 \mathrm{H}$ results. Based on the gene ontology analysis, Dnaja1 is predicted to participate in stress response while Gabra4 is involved in the system development process. The discovery of new host proteins that interact with ROP16 of $T$. gondii will help us to further investigate the functions of this effector proteins during $T$. gondii infection.

Keywords: Toxoplasma gondii, ROP16, protein interaction, Dnaja1, Gabra4, yeast two-hybrid

\footnotetext{
Abbreviations: cDNA, complementary deoxyribonucleic acid; Dnaja1, DnaJ heat shock protein family member A1; Gabra4, gamma-aminobutyric acid A receptor, subunit alpha 4; GO, Gene Ontology; GRAs, Dense granule proteins; HIV, Human immunodeficiency virus; IRG, immunity-related GTPases; NCBI, National Center for Biotechnology Information; PCR, Polymerase chain reaction; ROPs, Rhoptry proteins; STAT, signal transducer and activator of transcription; TgROP16, T. gondii rhoptry protein 16; Y2H, Yeast two-hybrid.
} 


\section{INTRODUCTION}

Toxoplasma gondii, as a model organism of Apicomplexa parasites, can infect all warm-blooded animals and result in toxoplasmosis worldwide (Montoya and Liesenfeld, 2004; Szabo and Finney, 2017). It's estimated that one third of the world population has been infected (Tenter et al., 2000; Montoya and Liesenfeld, 2004). As an opportunistic pathogen, it can cause severe disease in immunocompromised patients (Zhou et al., 2011; Zhang N.Z. et al., 2015). Latest studies show that people with HIV infection have a very high burden of $T$. gondii infection worldwide (Wang et al., 2017). As such, T. gondii is a big threat to the health of humans and animals (Jiang et al., 2015).

Over the last two decades, significant progress has been made in understanding the pathogenesis of T. gondii. Upon invasion, T. gondii parasites modify host cells intensively to make them a cozy home for their proliferation using various types of secretory proteins (Koshy et al., 2012). GRA and ROP proteins released from dense granules and rhoptries are the major parasite proteins to manipulate host gene transcription and protein expression. These include ROP5, ROP16, ROP18, and GRA15 etc (Hunter and Sibley, 2012; Cheeseman and Weitzman, 2015). ROPs play vital roles in mediating parasite-host interactions. Studied have found that ROP5, along with ROP17 and ROP18, controls virulence by blocking IFN- $\gamma$ activated autonomous immune clearance mechanisms in infected host cells (Behnke et al., 2012; Etheridge et al., 2014). ROP5, ROP18, and ROP17 form complex structures on the parasitophorous vacuole membrane (PVM) to phosphorylate IRG and prevent their accumulation on the PVM (Niedelman et al., 2012). Rhoptry protein ROP16, on the other hand, can directly localize to host cell nucleus and activate the host STAT signaling through phosphorylation of STAT3 and STAT6 (Yamamoto et al., 2009; Ong et al., 2010; Butcher et al., 2011; Hunter and Sibley, 2012), leading to prolonged activation of these two transcription factors and subsequent upregulation of interleukin-4 (IL-4) to antagonize IL-12 induction (Butcher et al., 2011; Hunter and Sibley, 2012). Further study have shown that ROP16 of type I and type III strains could sustain the STAT activation response but not the type II strain (Saeij et al., 2007). Moreover, ROP16 immunization could trigger a strong humoral and cellular responses against $T$. gondii (Yuan et al., 2011) and promote host resistance to oral infection and intestinal inflammation in mice, in the context of endogenous GRA15 expression (Jensen et al., 2013). These studies suggest that T. gondii ROP16 is an important virulence factor participating in modulation of host gene transcription and protein expression. However, besides STAT3 and STAT6, the host proteins modulated by TgROP 16 are currently unknown.

Yeast hybrid systems are widely used for the detection of protein-protein and protein-small molecule interactions. Among various yeast hybrid systems, $\mathrm{Y} 2 \mathrm{H}$ is the most commonly practiced, to detect protein-protein interactions and to screen complex genetic libraries for the identification of interacting partners for bait proteins (Legrain and Rain, 2014). It can also be modified to study the interactions of multiple proteins by reformative system (Pause et al., 1999). Many studies have used this tool to discover host proteins that interact with
T. gondii secretory proteins, which include TgMIC2, TgROP18, TgGRA15 etc (Cheng et al., 2012; Wang et al., 2014; Liu et al., 2017).

To further identify host proteins interacting with TgROP16, we use $\mathrm{Y} 2 \mathrm{H}$ method to screen a mouse brain cDNA library. Through this approach, we identified two mouse proteins, Dnaja1 and Gabra4, to interact with the Predomain of TgROP16, which forms new basis to elucidate the functions and mechanisms of ROP16 more comprehensively.

\section{MATERIALS AND METHODS}

\section{Parasite and Cell Lines}

Toxoplasma gondii type I strain RH was propagated in Human Foreskin Fibroblast (HFF) cells. Tachyzoites were harvested and purified by filtration through $3 \mu \mathrm{m}$ membranes (Whatman Inc., United States) before total RNA extraction. Seven-weekold female Kunming mice, were purchased from the Center for Disease Control (CDC) of Hubei Province in China for mice brain tissue RNA extraction. HEK293T cells were cultured in RPMI 1640 medium (Hyclone, United States) supplemented with $10 \%$ FBS (Gibco, United States) and 1\% penicillin/streptomycin (Gibco, United States).

\section{Plasmid Construction}

Total RNA was extracted from purified RH tachyzoites using TRIzol Reagent (Invitrogen, United States) according to manufacturer's instructions. Then one microgram of total RNA was reverse transcribed to cDNA using the ReverTra Ace reverse transcription kit (TOYOBO, Japan). The bait plasmids expressing different parts of TgROP16 were constructed by cloning one of the following three fragments of ROP16 into the pGBKT7 vector: the $2055 \mathrm{bp}$ fragment encoding the full length mature ROP16 (with signal peptide removed, 684 amino acids, from amino acid R24 to M707), the 1056 bp fragment encoding the Predomain (352 amino acids, from amino acid R24 to A375) and the $999 \mathrm{bp}$ fragment encoding the Kinase domain (332 amino acids, from amino acid L376 to M707). Using the cDNA above as templates, these three fragments were PCR amplified using specific primers mROP16-F (5'-GAATTCCGATACATGTCGTTTGAGGAAGC) and mROP16-R (5'-GTCGACGCTACATCCGATGTGAAGA AAG) for full length mature ROP16; ROP16-Pre-F (5'-GAATTCCGATACATGTCGTTTGAG) and ROP16-Pre-R (5'-GTCGACAGCGATCGGCACT) for the Predomain; ROP16Kinase-F (5'-GAATTCCTATACAATCGTGGGCAC) and ROP16-Kinase-R (5'-GTCGACCTACATCCGATGTGAAGA) for the Kinase domain. These PCR products were then digested with EcoRI and SalI, and then ligated into similarly digested pGBKT7.

For co-immunoprecipitation (Co-IP) assays, the three fragments of ROP16 mentioned above were tagged with Myc epitope and cloned into the pcDNA3.1 vector. The primers used to amplify these fragments for cloning were listed as follow: pCDNA3.1-ROP16-F (5'-GGAATTC ATGGAGGAGCAGAAGCTGATCTCAGAGGAGGACCTGCG 
ATACATGTCGTTTGAGGA) and pCDNA3.1-ROP16-R (5'CCGCTCGAGCTACATCCGATGTGAAGAAAGTT) for Myc tagged full length mature ROP16; pCDNA3.1-ROP16-F and pCDNA3.1-ROP16-Pre-R (5'-CCGCTCGAGCTAAG CGATCGGCACTCCGTTGG) for Myc tagged Predomain of ROP16; pCDNA3.1-ROP16-Kinase-F (5'GGAATTCATGGAGGAGCAGAAGCTGATCTCAGAGGAGG ACCTGCTATACAATCGTGGGCACCT) and pCDNA3.1ROP16-R for Myc tagged Kinase domain of ROP16. The corresponding PCR products were digested with EcoRI and XhoI sites, and cloned into PCDNA3.1 vector.

To clone the Dnajal gene from mouse, total RNA was extracted from mouse brain and reverse-transcribed into cDNA as above. Subsequently, the Dnajal gene was amplified from cDNA using Dnaja1-F (5'-GCTAGCGCTACCGGACTC AGATGGGCAAGGACTACTATCA) and Dnaja1-R (5'-TC CTCGCCCTTGCT CACCATTATGGGAAGAACCTGCTCCA) as primers, and was cloned into pEGFP-N1 using ClonExpress homologous recombinase (Vazyme Biotech, Co. Ltd, China). The linearized vector for such cloning was generated by amplification of pEGFP-N1 using primers pEGFP-N1-F (ATGGTGAGCAAGGGCGAGGA) and pEGFP-N1-R (CTG AGTCCGGTAGCGCTAGC).

\section{Autoactivation and Toxicity Test}

To test autoactivation and toxicity of bait protein TgROP16 in yeasts, bait plasmids containing the above mentioned three fragments of ROP16 were individually transformed into Saccharomyces cerevisiae strain $\mathrm{Y} 2 \mathrm{H}$ Gold according to the Yeastmaker Yeast Transformation System 2 User Manual (Clontech Laboratories, Inc., Mountain View, CA, United States). Transformants were then plated onto agar plates containing appropriate selection media, including $\mathrm{SD} /-\mathrm{Trp}, \mathrm{SD} /-\mathrm{Trp} / \mathrm{X}$ $(40 \mu \mathrm{g} / \mathrm{ml} \mathrm{X}-\alpha-\mathrm{Gal})$ or SD/-Trp/X/A $(40 \mu \mathrm{g} / \mathrm{ml} \mathrm{X- \alpha -Gal} \mathrm{and}$ $125 \mathrm{ng} / \mathrm{ml}$ Aureobasidin A). The plates were incubated at $30^{\circ} \mathrm{C}$ for 3 days and the growth (color and size) of transformants were recorded. The empty vector pGBKT7 was included as a control

\section{Western Blotting}

To check the expression of ROP16 in yeasts, Y2H Gold cells transformed with pGBKT7-mROP16 (a single clone) were inoculated into $\mathrm{SD} /$-Trp broth and cultured until $\mathrm{OD}_{600} \approx 0.6$. Then total proteins were extracted using the Urea/SDS method (Printen and Sprague, 1994; Wang et al., 2014) and subsequently separated on $12 \%$ SDS-PAGE gels and transferred onto PVDF membrane (Millipore, United States). Expression of ROP16 was probed with a mouse anti-Myc antibody (Medical and Biological Laboratories Co. Ltd., Japan), followed by an HRP-conjugated goat anti-mouse secondary antibody (Beyotime Institude of Biotechnology, China). The blot was developed using the direct ECL chemiluminescent method (GE Healthcare, United States).

\section{Y2H Screen}

Saccharomyces cerevisiae Y2H Gold and Y187 strains, as well as the medium were purchased from Clontech Co. (Mountain View, United States) for Y2H screen. The Mate \& Plate Universal Mouse (Normalized) cDNA library (Clontech, Mountain View,
United States) was cloned into the pGADT7-RecAB vector and expressed in Y187 yeast cells. To screen host proteins interacting with ROP16, Y2H Gold cells expressing ROP16 and Y187 cell expressing mouse proteins were mixed to mate for $20-24 \mathrm{~h}$ at $30^{\circ} \mathrm{C}$. Subsequently the mated cultures were plated onto SD/-Leu/-Trp plates supplemented with $40 \mu \mathrm{g} / \mathrm{ml} \mathrm{X- \alpha -Gal}$ and $125 \mathrm{ng} / \mathrm{ml}$ Aureobasidin $\mathrm{A}(\mathrm{DDO} / \mathrm{X} / \mathrm{A})$ and incubated at $30^{\circ} \mathrm{C}$ for 3 days, part of the mated cultures were also plated on $\mathrm{SD} /$-Trp, SD/-Leu, SD/-Trp/-Leu (DDO) plates to calculate the number of clones and the mating efficiency according to manufacturer's instructions. Afterwards, blue colonies were picked up and tested on higher stringency SD/-Ade/-His/-Leu/$\operatorname{Trp} / \mathrm{X}-\alpha-\mathrm{Gal} / \mathrm{AbA}(\mathrm{QDO} / \mathrm{X} / \mathrm{A})$ plates.

To identify the genes in positive hits, prey plasmids in positive hits were extracted using TIANprep yeast plasmid DNA kit (TIANGEN Co. LTD., Beijing, China), then sequenced by $\mathrm{T} 7$ primer and $3^{\prime} \mathrm{AD}$ primer. Sequencing results were blasted with NCBI databases to obtain gene information. Meanwhile, according to GO Classifications, molecular function and biological process of the prey genes were performed by searching the Mouse Genome Informatics (MGI) database ${ }^{1}$ to further estimate the function of the mouse proteins.

\section{Verification of Protein Interactions by $\mathrm{Y} 2 \mathrm{H}$}

To further confirm the potential positive hits, bait plasmids containing ROP16 and prey plasmids were transformed into $\mathrm{Y} 2 \mathrm{H}$ Gold and Y187 strains, respectively, according to manufacturer's instructions for small-scale transformation. Transformants were cultured on $\mathrm{SD} /$ - Trp and $\mathrm{SD} /$-Leu plates for 3 days at $30^{\circ} \mathrm{C}$ and single clones picked from each plate were used for mating. Mated heterozygotes were spread on SD/-Leu/-Trp/X- $\alpha-G a l$ (DDO/X) and $\mathrm{QDO} / \mathrm{X} / \mathrm{A}$ plates and grown for $3-5$ days at $30^{\circ} \mathrm{C}$. The empty bait vector pGBKT7 without ROP16 was used as a negative control.

To study the interaction between the prey protein and different domains of TgROP16, different fragments of ROP16 cloned in pGBKT7 and prey plasmids were transformed into $\mathrm{Y} 2 \mathrm{H}$ Gold and Y187 strain, respectively. Singles clones were mated and tested as above.

\section{Co-immunoprecipitation}

To verify the identified protein-protein interactions in vivo, plasmids pcDNA3.1 containing different parts of ROP16 and prey plasmid pEGFP-N1 containing mouse gene Dnaja1 were co-transfected into HEK293T cell for Co-IP assay. Bait and prey plasmids were mixed at the ratio of $1: 1$ and transfected into HEK293T cells using Lipofectamine 2000 (Invitrogen, United States) according to the manufacturer's instructions. Empty vectors were included as controls. The tranformants were grown for 28-36 h, harvested and lysed by ultrasonication in RIPA buffer (Beyotime Institude of Biotechnology, China) containing $1 \mathrm{mM}$ phenylmethylsulfonyl fluoride (Sigma-Aldrich Co. LLC., United States) and protease inhibitor cocktail (SigmaAldrich Co. LLC., United States). Supernatants of cell lysates

\footnotetext{
${ }^{1}$ http://www.informatics.jax.org/
} 
were incubated with mouse anti-Myc tag antibody (Medical and Biological Laboratories Co. Ltd., Japan) overnight, followed by Protein $\mathrm{G}$ agarose beads (Beyotime Institude of Biotechnology, China) incubation at $4^{\circ} \mathrm{C}$ for $6 \mathrm{~h}$. Subsequently, the agarose beads were washed with RIPA buffer and proteins bound to agarose beads were solubilized in $1 \times$ SDS-sample buffer containing dithiothreitol and boiled for $10 \mathrm{~min}$. Using Western blotting method, ROP16 was detected by mouse anti-Myc tag antibody and the bound Dnajal were detected by mouse anti-GFP (Proteintech Group Inc., United States). GAPDH detected by a mouse monoclonal antibody (Proteintech Group Inc., United States) was included as a loading control.

\section{RESULTS}

\section{Autoactivation, Toxicity, and Expression of ROP16 Baits in Y2H Gold Strain}

To screen for mouse proteins interacting with $T$. gondii ROP16, three different fragments of ROP16, including the mature full length ROP16 without signal peptide (mROP16), the Predomain (ROP16-Predomain) and the Kinase domain (ROP16-Kinase) (Figures 1A,B) were amplified from cDNA of $\mathrm{RH}$ tachyzoites and cloned into the pGBKT7 vector. These ROP16 expressing constructs were individually introduced into yeast cells and used as baits for two-hybrid screens. Before the screen, the autoactivation and toxicity of ROP16 in yeasts were estimated. To do that, bait plasmids expressing mROP16, ROP16Predomain, ROP16-Kinase domain were transformed into $\mathrm{Y} 2 \mathrm{H}$ Gold yeast cells, respectively. Transformants were grown on $\mathrm{SD} /$-Trp, SD/-Trp/X, or SD/-Trp/X/A plates (Figure 1D). Toxicity was indicated by poor growth on SD/-Trp plates, whereas autoactivation was determined by growth of colonies on SD/-Trp/X/A plates and blue colonies on SD/-Trp/X plates. Compared with the empty vector pGBKT7 control, all bait plasmids transformed yeast cells were growing well on SD/-Trp plates. Colonies on $\mathrm{SD} /$-Trp/X plates were white or orange colored instead of blue. No colony growth on SD/-Trp/X/A plates was observed (Figure 1D). Taken together, these data indicated that no obvious autoactivation and toxicity were detected for ROP16 baits.

To further check the expression of ROP16 bait in yeasts, $\mathrm{Y} 2 \mathrm{H}$ Gold cells transformed with mROP16 or the empty vector were subject to Western blot analysis. Total proteins were extracted from transformed cells by the Urea/SDS method. Western blotting analysis was performed using a Myc-tag antibody. The results indicated that $\mathrm{mROP} 16$ and control protein DNA-BD were expressed successfully in Y2H Gold cells (Figure 1C). The molecular weight of the mROP16 bait protein was approximately $111 \mathrm{kDa}$ composed of mROP16 protein $(90 \mathrm{kDa})$ fused to the DNA-BD protein $(21 \mathrm{kDa})$, which was consistent with what was detected on the Western blot.

\section{Y2H Screen}

To screen the host proteins that interact with TgROP16, Y187 yeast cells expressing a mouse cDNA library were used to mate with Y2H Gold cells expressing mROP16, as described in Section
"Materials and Methods". According to the manufacturer's instruction, mating efficiency was calculated to be $13.3 \%$, while $4.6 \times 10^{7}$ viable diploid yeast cells were screened with proper controls (Figures 2A,B). From the colonies grown on DDO/X/A plates, thirty blue clones were selected and tested on higher stringency QDO/X/A plates. Two clones (Clones \#9 and \#19) out of thirty still maintained blue (Figure 2C) while the other 28 failed to give blue colonies, indicating that two potential positive hits were obtained.

\section{Identification of Mouse Genes in Positive Hits}

Plasmids in the two positive clones were extracted and PCR amplified using the $\mathrm{T} 7$ primer and $3^{\prime} \mathrm{AD}$ primer to estimate the sizes of inserts. Gels electrophoresis results indicated that clone \#9 contained a $1.5 \mathrm{~Kb}$ insert, whereas clone \#19 had an insert of $2.1 \mathrm{~Kb}$ (Figure 2D). Further sequencing of the inserts and Blast analysis showed that clone \#9 contained the Dnaja1 (AB028273) gene and clone \#19 contained the Gabra4 (NM_010251) gene. According to GO classification, Dnajal likely participates in biological processes (cell death, establishment of localization, protein metabolic process, and response to stimulus) and performs molecular function like carbohydrate derivative binding, enzyme regulation, and receptor binding. Gabra4, on the other hand, may be involved in system development as a receptor or a transporter.

\section{Verification of Identified Hits by $\mathbf{Y} \mathbf{2 H}$}

To further verify these two clones, prey plasmids extracted from them were individually transformed into Y187 cells. Subsequently transformants were used to mate with $\mathrm{Y} 2 \mathrm{H}$ Gold cells expressing mROP16 and plated on DDO/X and QDO/X/A indication plates. Growth of blue colonies on these plates implied corresponding protein interactions. As shown in Figure 2E, both Dnaja1 and Gabra4 did interact with full length ROP16 as blue colonies were observed on both DDO/X and QDO/X/A plates.

To examine the interactions between Dnaja1/Gabra4 and each domain of ROP16, ROP16-Predomain and ROP16-Kinase domain expressing plasmids were transformed into $\mathrm{Y} 2 \mathrm{H}$ Gold cells, respectively, and mated with Dnaja1 or Gabra4 expressing Y187 cells. Mated cultures were plated on the above-mentioned indication plates. The results showed that Dnajal mainly interacted with full length ROP16, as blue colonies were only observed during mROP16 mating (Figure 2E). Dnajal also interacted with the Predomain of ROP16. But the interaction was probably not as strong as with full length ROP16, since less blue colonies were observed during ROP16-Predomain mating (Figure 2E). Gabra4, on the other hand, interacted with both full length and the Predomain of ROP16 (Figure 2E).

\section{Validation of Protein Interactions by Co-IP}

In order to verify the interactions between ROP16 and host proteins in vivo, Dnajal was chose to be co-expressed with different domains of ROP16 in HEK293T cells and assess their interactions by Co-IP. First, expression of each of these proteins 
A

$$
\begin{aligned}
& \text { ROP16 full length } \\
& \text { pGBKT7-mROP16 } \\
& \text { aa:24-707 }
\end{aligned}
$$

pGBKT7-ROP16 Predomain

aa:24-375

pGBKT7-ROP16 Kinase domain

aa:376-707

B
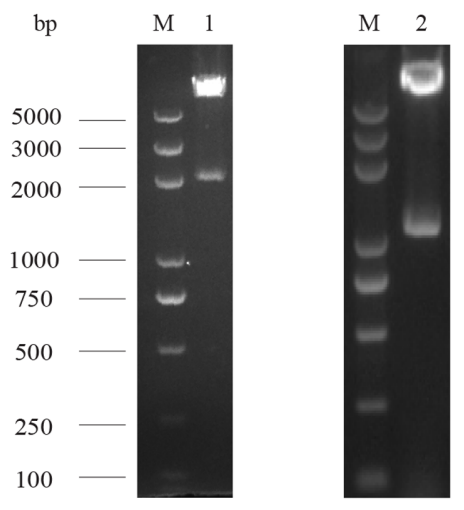
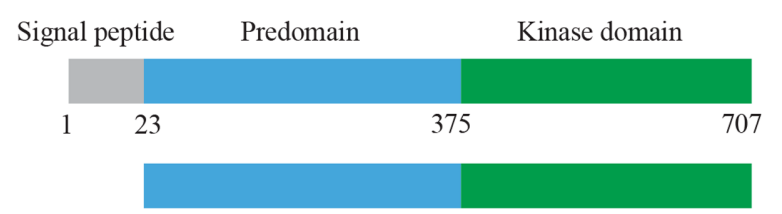

24

375

707

24

375
707

C

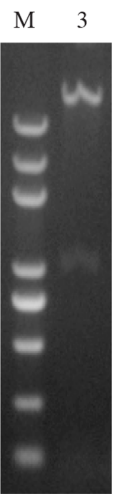

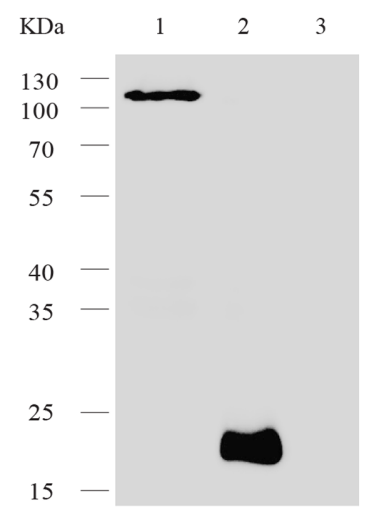

D

$\mathrm{SD} /-\mathrm{Trp}$

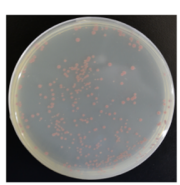

pGBKT7-mROP16

pGBKT7

pGBKT7-ROP16-Predomain

pGBKT7-ROP16-Kinase domain
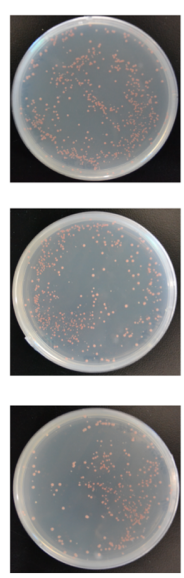

$\mathrm{SD} /-\operatorname{Trp} / \mathrm{X}$
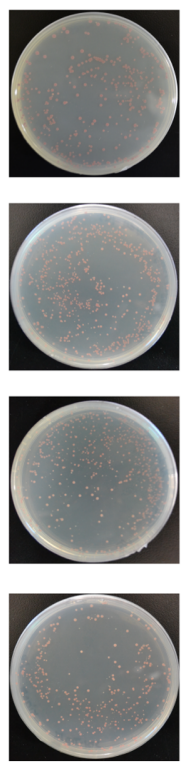

$\mathrm{SD} /-\operatorname{Trp} / \mathrm{X} / \mathrm{A}$
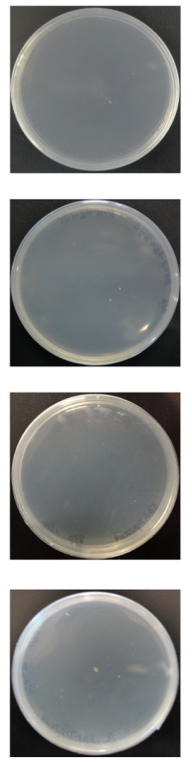

FIGURE 1 | Autoactivation, toxicity and expression tests for ROP16 expressing bait plasmids in yeast cells. (A) Schematic illustration of ROP16 full length and different domains of ROP16, mROP16, ROP16-Predomain, and ROP16-Kinase domain. (B) Agarose gel electrophoresis of bait plasmids digested with EcoRI and Sall. M: DNA marker 2K plus; Lane 1: pGBKT7-mROP16; Lane 2: pGBKT7-ROP16-Predomain; Lane 3: pGBKT7-ROP16-Kinase domain. (C) Western blotting analysis of ROP16 bait expression in yeast cell detected by mouse anti-Myc antibodies. Lane 1: Y2H Gold/pGBKT7-mROP16; Lane 2: Y2H Gold/pGBKT7; lane 3: Y2H Gold cell. (D) Detection of autoactivation and toxicity of bait plasmids with SD/-Trp, SD/-Trp/X, and SD/-Trp/X/A indication plates. 


\section{A}

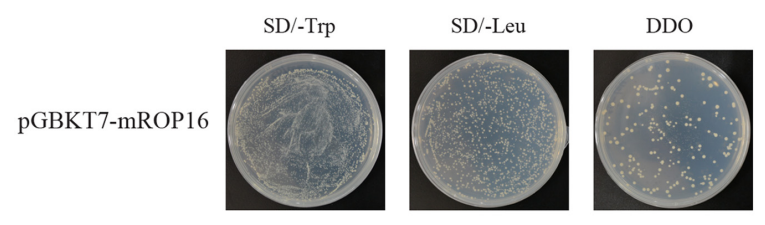

C
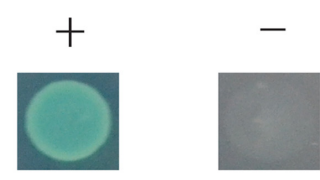

Clone 9

Clone 19
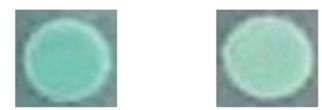

pGBKT7

$\mathbf{E}$
B

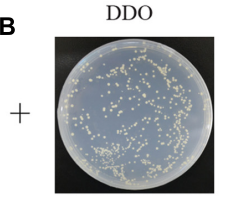

DDO/X/A

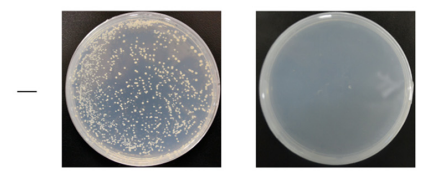

D

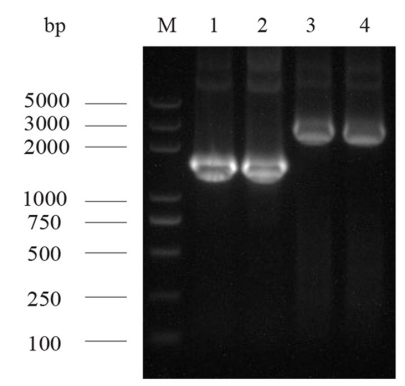

Gabra4

DDO/X

QDO/X/A
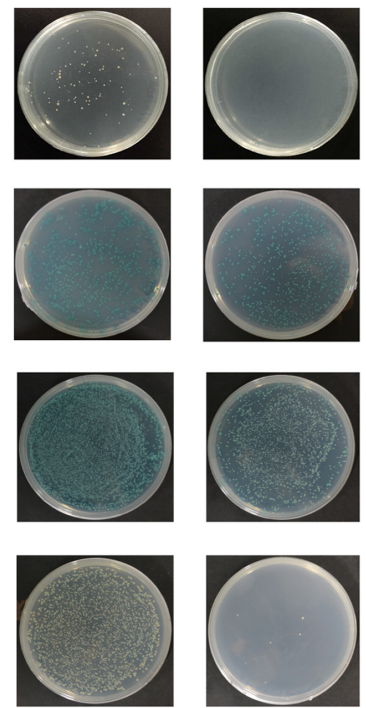

FIGURE 2 | Screen of mouse proteins interacting with mROP16 using yeast two-hybrid $(Y 2 H)$. (A) Determination of mating efficiency. Equal amount of mated cultures (Y2H Gold/mROP16 + Y187/mouse cDNA library) were plated on SD/-Trp, SD/-Leu, and SD/-Trp/-Leu (DDO) plates. Mating efficiency was calculated by dividing the number of colonies on DDO plate by the number of colonies on SD/-Trp or SD/-Leu plates, whichever is less (In this example, there are less colonies on $\mathrm{SD}$ /-Leu plate than on SD/-Trp plate, therefore the number of colonies on SD/-Leu plate was used for mating efficiency calculation). (B) Growth of positive (+, pGBKT7-53 and pGADT7-T) and negative (-, pGBKT7-Lam and pGADT7-T) controls on Y2H DDO and DDO/X/A indication plates. (C) Growth of two positive clones (\#9 and \#19) on QDO/X/A plates, + and - are positive and negative control colonies, respectively. (D) PCR amplification of the inserts in positive clones. M: DNA Marker. Lane 1-2: Clone 9; Lane 3-4: Clone 19. (E) Interaction between the two hits and different domains of ROP16. Dnaja1 or Gabra4 expressing plasmids and different domains of ROP16 were transformed into $\mathrm{Y} 187$ and $\mathrm{Y} 2 \mathrm{H}$ Gold cells, respectively. Transformants were used to mate and grown on DDO/X and QDO/X/A plates to monitor protein interactions. 

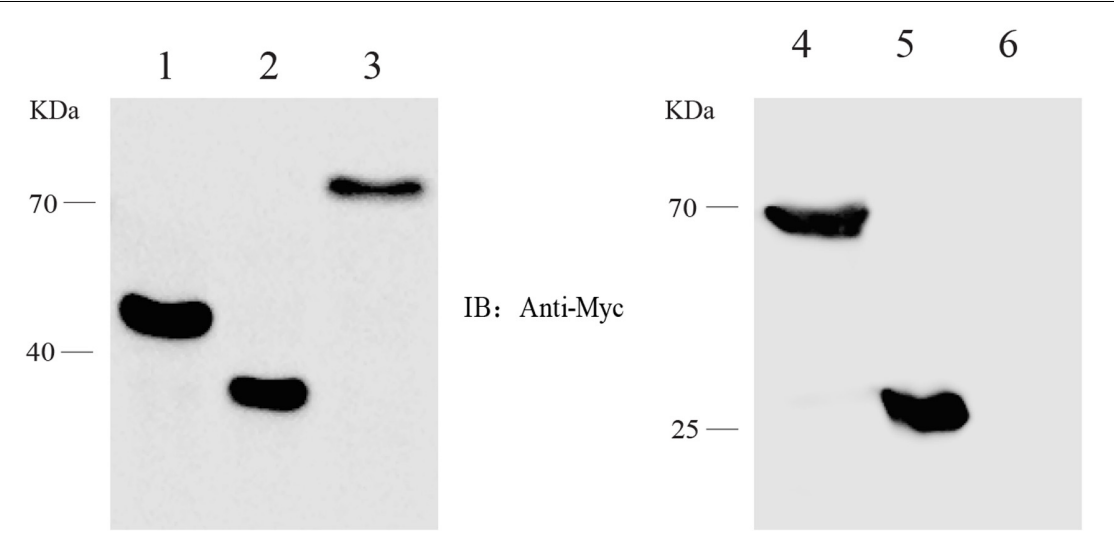

IB: Anti-GFP

FIGURE 3 | Expression of different parts of ROP16, as well as Dnaja1 in HEK293T cells. HEK293T cells transfected with ROP16 expressing plasmids or Dnaja1 expressing plasmids were subject to Western blotting analysis $24 \mathrm{~h}$ post transfection. ROP16 fragments expressed from pCDNA3.1 were detected by anti-Myc tag antibody and Dnaja1 expressed from pEGFP-N1 was detected by an anti-GFP monoclonal antibody. Lane 1: pCDNA3.1-ROP16-Predomain; Lane 2: pCDNA3.1-ROP16-Kinase domain; Lane 3: pCDNA3.1-mROP16, Lane 4: pEGFP-N1-Dnaja1; Lane 5: pEGFP-N1; Lane 6: empty HEK293T cell.

in HEK293T cells were examined. To do this, pCDNA3.1 containing different fragments of ROP16 was individually transfected into pCDNA3.1 and then transfected into HEK293T cells using Lipofectamine 2000, and expression of ROP16 fragments was determined by Western blotting using an anti-Myc antibody $24 \mathrm{~h}$ post transfection. Results in Figure 3 showed that all three fragments of ROP16 could be well expressed in HEK293T cells. Similarly, Dnajal was also expressed well in HEK293T cells as a GFR fusion (pEGFP-Dnaja1) and detected by anti-GFP monoclonal antibodies (Figure 3). To assess protein interactions by Co-IP, each of the ROP16 fragment expressing plasmids was mixed with pEGFP- Dnajal at the ratio of $1: 1$, and co-transfected into HEK293T cells. Cell lysates prepared from transfectants $24 \mathrm{~h}$ post transfection were immunoprecipitated with an anti-Myc antibody. The GFP-Dnajal protein bound to precipitated ROP16 was detected by an anti-GFP antibody. Consistent with the $\mathrm{Y} 2 \mathrm{H}$ results, GFP-Dnajal was co-precipitated with full length mature ROP16, as well as the Predomain of ROP16 (Figures 4A,B). Dnajal did not show obvious co-precipitation with the Kinase domain of ROP16, consistent with the lack of interaction of the two (Figure 4C). The interactions detected by Co-IP were likely to be specific, since negative controls using GFP alone or Myc tag alone did not give detectable Co-IP products (Figure 4). Together these results indicated that Dnajal was able to be interact with full length, as well as Predomain of ROP16.

\section{DISCUSSION}

Previous studies have reported that T. gondii rhoptry protein ROP16 was involved in the activation of host STAT signaling pathway when it reached host cell nuclei (Hunter and Sibley, 2012). STAT3 and STAT6 were shown to be phosphorylated by ROP16 (Yamamoto et al., 2009; Ong et al., 2010; Butcher et al., 2011), but different alleles of ROP16 exhibited different capacities in activating STAT signaling (Saeij et al., 2007). Of the three clonal strain types (type I/II/III), all strains were able to induce STAT3 and STAT6 activation initially, but only type I and type III strains sustained this response and type II strains did not. In this study, a high throughput $\mathrm{Y} 2 \mathrm{H}$ screen was used to search for host proteins interacting with ROP16 of type I strain RH. Two proteins, Dnaja1 (AB028273) and Gabra4 (NM_010251) were identified from a mouse cDNA library to interact with ROP16. The interactions between Dnajal and different parts of ROP16 were further estimated by Co-IP and the results showed that the Predomain of ROP16 was the major domain to mediate such interactions. Co-IP was also tried to assess the interaction between Gabra4 and ROP16, however, no informative results were obtained because the expression of Gabra4 in HEK293T cells was below the detection limit. The reason for this low expression is currently unknown. Due to the limitations of the yeast two hybrid approach, our screen is probably not yet exhausted because known TgROP16 interactors such as STAT3 and STAT6 were not identified. In addition to the common limitations of yeast two hybrid screen, another important factor that determines the outcome of our screen is the host cDNA library. The one used in our screen is derived from mouse brain, which may have different gene expression patterns than other tissues. This may partially explain why only two proteins were identified from our screen. In this regard, further work is needed to screen libraries derived from other tissues to estimate the overall interactions between ROP16 and host proteins.

Dnaja1, a member of the HSP40s family proteins, is highly conserved (greater than 95\% sequence similarity) in most vertebrates (Orthwein et al., 2012). Many proteins that interacted with Dnajal have been identified. In the presence of environmental stress, human Dnajal activates a DnaK protein through forming a complex with it, to suppress the JNK pathway and the hyperphosphorylation of c-Jun (Stark et al., 2014). Down-regulation of Dnajal in pancreatic cancer cells probably reduced the activity of DnaK even under stress conditions, which promoted the occurrence and progression of cancer due to cell proliferation (Stark et al., 2014). Another 


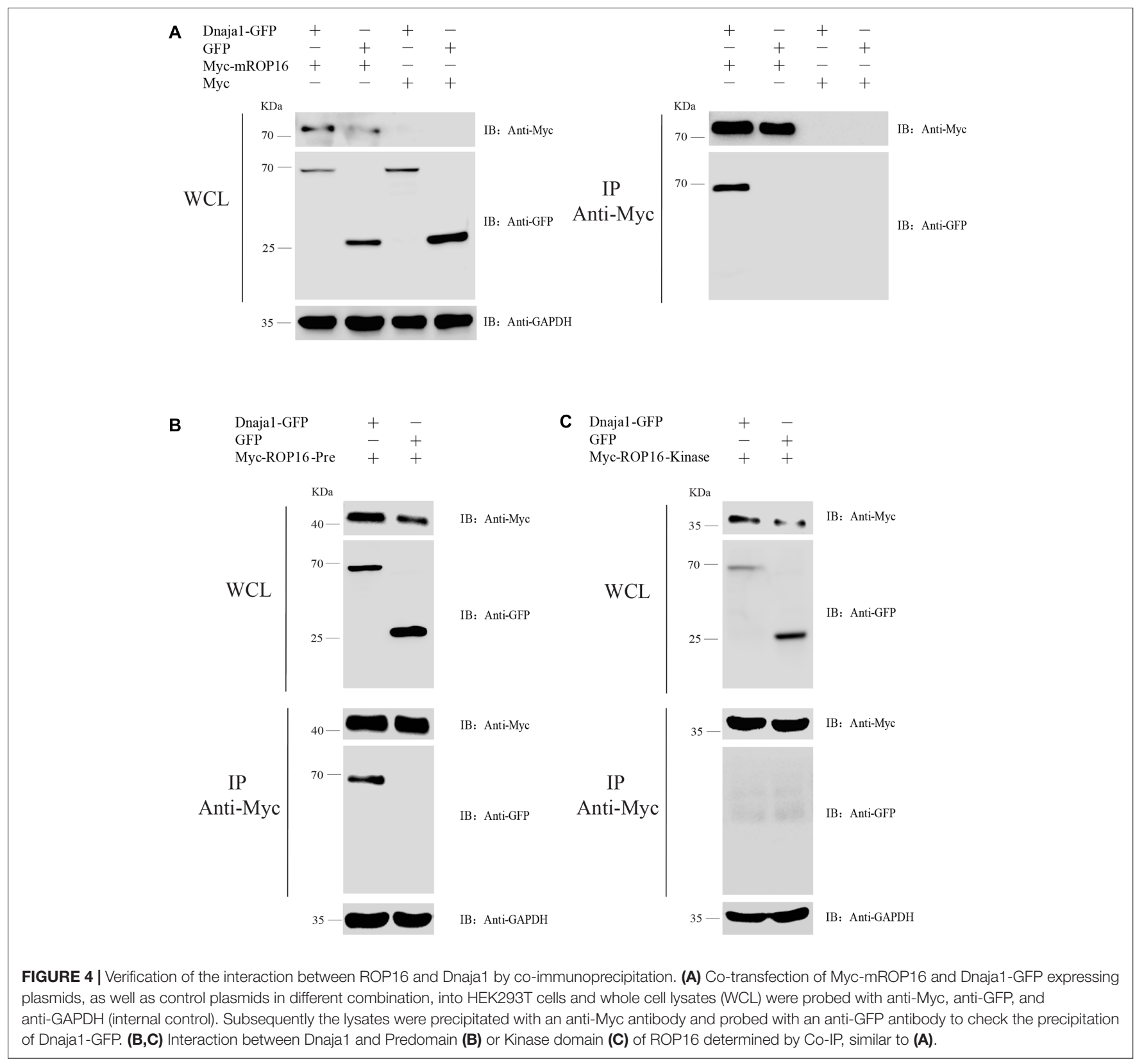

study demonstrated that activation-induced deaminase (AID) interacted with Dnaja1 and Dnaja2 in vitro, but only Dnaja1 over-expression increased AID levels and biological activities, while depletion of Dnaja1 reduced AID levels in cell lines (Orthwein et al., 2012). Previous studies have revealed that AID contributed to antibody-mediated autoimmune diseases (Zaheen and Martin, 2011) as well as to cancer (Okazaki et al., 2003; Pasqualucci et al., 2008). Thus, Dnajal plays a nonredundant role in the stabilization of AID and balancing immunity and cancer development indirectly (Orthwein et al., 2012). Moreover, Dnajal was verified as a novel substrate to interact with histone deacetylase 6 (HDAC6) by quantitative proteomic analyses (Zhang L. et al., 2015), while HDAC6 played a role to initiate proper impaired immune response with the model of HDAC6-deficient mouse (Zhang et al., 2008). With the results from this work and previous studies, we speculate that ROP16-Dnaja1 complex may participate in immune response modulation. Further work is needed to dissect the biological significance of this interaction.

Gabra4, which encodes the $\alpha 4$ subunit of gammaaminobutyric acid A receptor, belong to alcohol-regulated genes (ARGs) that show remarkable plasticity in response to alcohol (Suryanarayanan et al., 2011). Previous studies indicated that Gabra was considered to be the primary molecular target of injectable anesthetics (Iyer et al., 2014). It is rapidly up-regulated by acute alcohol exposure and down-regulated in cortical neurons during alcohol withdrawal. Gabra4 was regulated by specific microRNAs (Bekdash and Harrison, 2015), as well as 
protein kinases (PK) like PKA and PKC (Carlson et al., 2016). Further study have shown that Gabra4 played a critical role in trafficking of the $\delta$ subunit to the neuronal surface where both were major mediators of tonic inhibition in the thalamus (Peng et al., 2014). The levels of Gabra4 mRNA and protein were elevated in spontaneous seizures (Grabenstatter et al., 2014). According to the role of Gabra4 in stress response and nervous system activity, ROP16 may affect these processes through interacting with Gabra4.

As a protein kinase, ROP16 is composed of a Predomain and Kinase domian with a signal peptide at the $\mathrm{N}$-terminus (Figure 1A). Previous studies demonstrated that type I and type III ROP16 could modify STAT3 by phosphorylation, but type II ROP16 could not. A single polymorphic amino acid (L503S) in the Kinase domain of ROP16 determined STAT3 activation (Yamamoto et al., 2009). On the other hand, little information is available regarding the function of the Predomain. In this study, the interactions between ROP16-Predomain and Dnaja1 was identified through $\mathrm{Y} 2 \mathrm{H}$ and Co-IP, indicating important roles of the Predomian of ROP16. Further studies are required to explore the exact functions of ROP16-Predomain during Toxoplasma infection.

In summary, two mouse proteins, Dnaja1 and Gabra4 were identified to interact with TgROP16 by $\mathrm{Y} 2 \mathrm{H}$ screen. The interaction between ROP16 and Dnajal was further verified by $\mathrm{Y} 2 \mathrm{H}$, as well as Co-IP methods. These results

\section{REFERENCES}

Behnke, M. S., Fentress, S. J., Mashayekhi, M., Li, L. X., Taylor, G. A., and Sibley, L. D. (2012). The polymorphic pseudokinase ROP5 controls virulence in Toxoplasma gondii by regulating the active kinase ROP18. PLOS Pathog. 8:e1002992. doi: 10.1371/journal.ppat.1002992

Bekdash, R. A., and Harrison, N. L. (2015). Downregulation of Gabra4 expression during alcohol withdrawal is mediated by specific microRNAs in cultured mouse cortical neurons. Brain Behav. 5:e00355. doi: 10.1002/brb3.355

Butcher, B. A., Fox, B. A., Rommereim, L. M., Kim, S. G., Maurer, K. J., Yarovinsky, F., et al. (2011). Toxoplasma gondii rhoptry kinase ROP16 activates STAT3 and STAT6 resulting in cytokine inhibition and arginase-1-dependent growth control. PLOS Pathog. 7:e1002236. doi: 10.1371/journal.ppat.1002236

Carlson, S. L., Bohnsack, J. P., and Morrow, A. L. (2016). Ethanol regulation of synaptic GABAA alpha4 receptors is prevented by protein kinase a activation. J. Pharmacol. Exp. Ther. 357, 10-16. doi: 10.1124/jpet.115.230417

Cheeseman, K., and Weitzman, J. B. (2015). Host-parasite interactions: an intimate epigenetic relationship. Cell. Microbiol. 17, 1121-1132. doi: 10.1111/cmi.12471

Cheng, L., Chen, Y., Chen, L., Shen, Y., Shen, J., An, R., et al. (2012). Interactions between the ROP18 kinase and host cell proteins that aid in the parasitism of Toxoplasma gondii. Acta Trop. 122, 255-260. doi: 10.1016/j.actatropica.2012. 02.001

Etheridge, R. D., Alaganan, A., Tang, K., Lou, H. J., Turk, B. E., and Sibley, L. D. (2014). The Toxoplasma pseudokinase ROP5 forms complexes with ROP18 and ROP17 kinases that synergize to control acute virulence in mice. Cell Host Microbe 15, 537-550. doi: 10.1016/j.chom.2014.04.002

Grabenstatter, H. L., Cogswell, M., Cruz Del Angel, Y., Carlsen, J., Gonzalez, M. I., Raol, Y. H., et al. (2014). Effect of spontaneous seizures on GABAA receptor alpha4 subunit expression in an animal model of temporal lobe epilepsy. Epilepsia 55, 1826-1833. doi: 10.1111/epi.12771

Hunter, C. A., and Sibley, L. D. (2012). Modulation of innate immunity by Toxoplasma gondii virulence effectors. Nat. Rev. Microbiol. 10, 766-778. doi: $10.1038 /$ nrmicro2858

Iyer, S. V., Chandra, D., and Homanics, G. E. (2014). GABAA-R alpha4 subunits are required for the low dose locomotor stimulatory effect of alphaxalone, but not provided important clues to further investigate the functions of TgROP16.

\section{AUTHOR CONTRIBUTIONS}

JZ conceived and designed the study. JZ and YZ revised the manuscript critically. MP performed the studies and wrote the manuscript. YW assisted in the yeast two-hybrid screen. LL, YS, and LH contributed to yeast mating and Co-IP assay. All authors read and approved the final manuscript.

\section{FUNDING}

This work was supported by National Natural Science Foundation of China (Grant \# 31372429), as well as the Fundamental Research Funds for the Central Universities in China (Project 2662015PY048).

\section{ACKNOWLEDGMENT}

The authors would like to acknowledge the helpful advices and comments from Drs. Bang Shen, Min Hu, Lan He, and Rui Fang during this study.

for several other behavioral responses to alphaxalone, etomidate or propofol. Neurochem. Res. 39, 1048-1056. doi: 10.1007/s11064-013-1148-3

Jensen, K. D., Hu, K., Whitmarsh, R. J., Hassan, M. A., Julien, L., Lu, D., et al. (2013). Toxoplasma gondii rhoptry 16 kinase promotes host resistance to oral infection and intestinal inflammation only in the context of the dense granule protein GRA15. Infect. Immun. 81, 2156-2167. doi: 10.1128/IAI. 01185-12

Jiang, C., Li, Z., Chen, P., and Chen, L. (2015). The seroprevalence of Toxoplasma gondii in Chinese population with cancer: a systematic review and metaanalysis. Medicine 94:e2274. doi: 10.1097/MD.0000000000002274

Koshy, A. A., Dietrich, H. K., Christian, D. A., Melehani, J. H., Shastri, A. J., Hunter, C. A., et al. (2012). Toxoplasma co-opts host cells it does not invade. PLOS Pathog. 8:e1002825. doi: 10.1371/journal.ppat.1002825

Legrain, P., and Rain, J. C. (2014). Twenty years of protein interaction studies for biological function deciphering. J. Proteomics 107, 93-97. doi: 10.1016/j.jprot. 2014.03.038

Liu, Q., Li, F. C., Elsheikha, H. M., Sun, M. M., and Zhu, X. Q. (2017). Identification of host proteins interacting with Toxoplasma gondii GRA15 (TgGRA15) by yeast two-hybrid system. Parasit. Vectors 10:1. doi: 10.1186/s13071-016-1943-1

Montoya, J. G., and Liesenfeld, O. (2004). Toxoplasmosis. Lancet 363, 1965-1976. doi: 10.1016/S0140-6736(04)16412-X

Niedelman, W., Gold, D. A., Rosowski, E. E., Sprokholt, J. K., Lim, D., Farid Arenas, A., et al. (2012). The rhoptry proteins ROP18 and ROP5 mediate Toxoplasma gondii evasion of the murine, but not the human, interferongamma response. PLOS Pathog. 8:e1002784. doi: 10.1371/journal.ppat.1002784

Okazaki, I. M., Hiai, H., Kakazu, N., Yamada, S., Muramatsu, M., Kinoshita, K., et al. (2003). Constitutive expression of AID leads to tumorigenesis. J. Exp. Med. 197, 1173-1181. doi: 10.1084/jem.20030275

Ong, Y. C., Reese, M. L., and Boothroyd, J. C. (2010). Toxoplasma rhoptry protein 16 (ROP16) subverts host function by direct tyrosine phosphorylation of STAT6. J. Biol. Chem. 285, 28731-28740. doi: 10.1074/jbc.M110. 112359

Orthwein, A., Zahn, A., Methot, S. P., Godin, D., Conticello, S. G., Terada, K., et al. (2012). Optimal functional levels of activation-induced deaminase specifically require the Hsp40 DnaJa1. EMBO J. 31, 679-691. doi: 10.1038/emboj.2011.417 
Pasqualucci, L., Bhagat, G., Jankovic, M., Compagno, M., Smith, P., Muramatsu, M., et al. (2008). AID is required for germinal center-derived lymphomagenesis. Nat. Genet. 40, 108-112. doi: 10.1038/ng.2007.35

Pause, A., Peterson, B., Schaffar, G., Stearman, R., and Klausner, R. D. (1999). Studying interactions of four proteins in the yeast two-hybrid system: structural resemblance of the $\mathrm{pVHL} /$ elongin BC/hCUL-2 complex with the ubiquitin ligase complex SKP1/cullin/F-box protein. Proc. Natl. Acad. Sci. U.S.A. 96, 9533-9538. doi: 10.1073/pnas.96.17.9533

Peng, Z., Zhang, N., Chandra, D., Homanics, G. E., Olsen, R. W., and Houser, C. R. (2014). Altered localization of the delta subunit of the GABAA receptor in the thalamus of alpha4 subunit knockout mice. Neurochem. Res. 39, 1104-1117. doi: 10.1007/s11064-013-1202-1

Printen, J. A., and Sprague, G. F. Jr. (1994). Protein-protein interactions in the yeast pheromone response pathway: ste $5 \mathrm{p}$ interacts with all members of the MAP kinase cascade. Genetics 138, 609-619.

Saeij, J. P., Coller, S., Boyle, J. P., Jerome, M. E., White, M. W., and Boothroyd, J. C. (2007). Toxoplasma co-opts host gene expression by injection of a polymorphic kinase homologue. Nature 445, 324-327. doi: 10.1038/nature 05395

Stark, J. L., Mehla, K., Chaika, N., Acton, T. B., Xiao, R., Singh, P. K., et al. (2014). Structure and function of human DnaJ homologue subfamily a member 1 (DNAJA1) and its relationship to pancreatic cancer. Biochemistry 53, 1360-1372. doi: 10.1021/bi401329a

Suryanarayanan, A., Liang, J., Meyer, E. M., Lindemeyer, A. K., Chandra, D., Homanics, G. E., et al. (2011). Subunit compensation and plasticity of synaptic GABA(A) receptors induced by ethanol in alpha4 subunit knockout mice. Front. Neurosci. 5:110. doi: 10.3389/fnins.2011.00110

Szabo, E. K., and Finney, C. A. (2017). Toxoplasma gondii: one organism, multiple models. Trends Parasitol. 33, 113-127. doi: 10.1016/j.pt.2016.11.007

Tenter, A. M., Heckeroth, A. R., and Weiss, L. M. (2000). Toxoplasma gondii: from animals to humans. Int. J. Parasitol. 30, 1217-1258. doi: 10.1016/S00207519(00)00124-7

Wang, Y., Fang, R., Yuan, Y., Hu, M., Zhou, Y., and Zhao, J. (2014). Identification of host proteins interacting with the integrin-like A domain of Toxoplasma gondii micronemal protein MIC2 by yeast-two-hybrid screening. Parasit. Vectors 7:543. doi: 10.1186/s13071-014-0543-1

Wang, Z. D., Wang, S. C., Liu, H. H., Ma, H. Y., Li, Z. Y., Wei, F., et al. (2017). Prevalence and burden of Toxoplasma gondii infection in HIV-infected people: a systematic review and meta-analysis. Lancet HIV 4, e177-e188. doi: 10.1016/ S2352-3018(17)30005-X

Yamamoto, M., Standley, D. M., Takashima, S., Saiga, H., Okuyama, M., Kayama, H., et al. (2009). A single polymorphic amino acid on Toxoplasma gondii kinase ROP16 determines the direct and strain-specific activation of Stat3. J. Exp. Med. 206, 2747-2760. doi: 10.1084/jem.20091703

Yuan, Z. G., Zhang, X. X., He, X. H., Petersen, E., Zhou, D. H., He, Y., et al. (2011). Protective immunity induced by Toxoplasma gondii rhoptry protein 16 against toxoplasmosis in mice. Clin. Vaccine Immunol. 18, 119-124. doi: 10.1128/CVI.00312-10

Zaheen, A., and Martin, A. (2011). Activation-induced cytidine deaminase and aberrant germinal center selection in the development of humoral autoimmunities. Am. J. Pathol. 178, 462-471. doi: 10.1016/j.ajpath.2010.09.044

Zhang, L., Liu, S., Liu, N., Zhang, Y., Liu, M., Li, D., et al. (2015). Proteomic identification and functional characterization of MYH9, Hsc70, and DNAJA1 as novel substrates of HDAC6 deacetylase activity. Protein Cell 6, 42-54. doi: 10.1007/s13238-014-0102-8

Zhang, N. Z., Wang, M., Xu, Y., Petersen, E., and Zhu, X. Q. (2015). Recent advances in developing vaccines against Toxoplasma gondii: an update. Expert Rev. Vaccines 14, 1609-1621. doi: 10.1586/14760584.2015.1098539

Zhang, Y., Kwon, S., Yamaguchi, T., Cubizolles, F., Rousseaux, S., Kneissel, M., et al. (2008). Mice lacking histone deacetylase 6 have hyperacetylated tubulin but are viable and develop normally. Mol. Cell. Biol. 28, 1688-1701. doi: 10.1128/MCB. 01154-06

Zhou, P., Chen, Z., Li, H. L., Zheng, H., He, S., Lin, R. Q., et al. (2011). Toxoplasma gondii infection in humans in China. Parasit. Vectors 4:165. doi: 10.1186/17563305-4- 165

Conflict of Interest Statement: The authors declare that the research was conducted in the absence of any commercial or financial relationships that could be construed as a potential conflict of interest.

Copyright (c) 2017 Pan, Zhou, Wang, Li, Song, Hou and Zhao. This is an open-access article distributed under the terms of the Creative Commons Attribution License (CC BY). The use, distribution or reproduction in other forums is permitted, provided the original author(s) or licensor are credited and that the original publication in this journal is cited, in accordance with accepted academic practice. No use, distribution or reproduction is permitted which does not comply with these terms. 